\title{
Estudio piloto de la postura de pie en deportistas
}

X. BORRÀS ${ }^{1}$, F. MARÍN ${ }^{1}$

${ }^{1}$ Sport Performance Analysis Research Group (SPARG)

UVic-UCC, Vic

\begin{abstract}
Resumen
El objetivo de este estudio piloto es el de analizar la postura de pie en deportistas de diferentes modalidades. Participaron 6 estudiantes masculinos y 1 estudiante femenina, no lesionados. Se tomaron 4 fotografias de los diferentes perfiles que fueron procesadas mediante un programa de análisis postural (SAPO, Sao Paulo). Las referencias anatómicas a digitalizar fueron señalizadas mediante 27 marcadores. Los resultados muestran que, de media, los sujetos presentan desviaciones posturales leves respecto a los valores de referencia, sobretodo destaca la desviación en la alineación vertical de la cabeza $\left(18^{\circ}\right)$. La metodología permite identificar las desviaciones posturales y aplicarla a estudios con un mayor número de sujetos. Esto podría permitir identificar patrones de postura considerados factores de riesgo para padecer una lesión. Así mismo se podría conocer si se producen modificaciones de la postura en función del tipo de modalidad deportiva practicada.
\end{abstract}

Palabras clave: Fotogrametría, análisis postural, deporte, postura de pie.

\begin{abstract}
The objective of this pilot study was to analyse the standing posture of physical active subjects, participating in different types of sport. Subjects were 6 male and 1 female, not injured. Photographs form the frontal (anterior and posterior) and sides (left and right) views were taken and processed by a specific postural analysis software (SAPO, Sao Paulo). 27 markers were placed on anatomical references. Results show that subjects present slight deviations from the reference values. Highlights the vertical head alienation $\left(18^{\circ}\right)$. In conclusion, it is possible to detect postural deviations through photogrammetry; this could allow the identification postural patterns considered injury risk factors. Likewise, it could be known if there are postural modifications due to the typology of sport and activity practised.
\end{abstract}

Keywords: Photogrammetry, postural analysis, sport, standing posture.

Correspondencia:

Xantal Borràs

E-mail: xantal.borras@uvic.cat

DOI: $10.5821 /$ sibb.25.1.5392 


\section{Introducción}

La postura se define como la manera de colocar los diferentes segmentos corporales. La alineación ideal es aquella que permite mantener el equilibrio con el mínimo coste energético, es decir, menos esfuerzo del sistema músculo-esquelético. En la postura ideal existe simetría entre los dos lados del cuerpo para el plano frontal. Des del plano sagital, una línea vertical tiene que pasar por el centro de masa de la persona y por la base de las orejas, centro articular de hombro, cadera, rodilla y justo por delante del maléolo externo del tobillo [1].

El objetivo de la observación de la postura es adquirir información sobre defectos visibles, déficits funcionales y anormalidades en la alienación [2]. Problemas posturales generalmente generan elevadas tensiones de las estructuras de sostén del sistema musculo-esquelético. Para la recuperación de la funcionalidad y mantenimiento o mejora de la postura se utilizan entrenamientos correctores y tratamientos fisioterapéuticos

La resonancia magnética en 3D y la tomografía computarizada en $4 \mathrm{D}$ son considerados los estándares de oro para la valoración de la postura, pero son métodos con un elevado coste económico. Los rayos X son otro sistema de valoración de la postura, sobretodo de la columna vertebral, pero debido a las radiaciones no puede ser utilizado de manera habitual en el ser humano [3]. La valoración de la postura mediante fotografías es utilizada como substitución, ya que es simple, económico y no invasivo [4].

Varios estudios han demostrado la fiabilidad y validez del método fotográfico, pero es imprescindible colocar correctamente los marcadores $[3,5,6,7,8]$.

El método de la valoración postural mediante fotografía se ha utilizado en niños [6, 9,10 ], adolescentes [11], estudiantes universitarios [12, 13], adultos jóvenes [7, 8] y adultos $[2,5]$. No obstante falta información postural en deportistas, sobre los efectos beneficiosos de la práctica de actividad física y las adaptaciones posturales a determinados deportes.

Rosário y col [13] utilizaron el método fotográfico para evaluar los efectos de diferentes tipos de estiramientos sobre la postura (estiramientos por segmento versus terapia de cadenas musculares) y observan que el efecto sobre la postura es mayor cuando se utiliza un método global de estiramiento. Sizato y col [14] lo utilizan para evaluar el efecto de 20 sesiones de Pilates sobre la postura en mujeres jóvenes $y$, aunque observan mejoras en la amplitud de movimiento concluyen que 20 sesiones no son suficientes para provocar adaptaciones posturales. Munivrana y col [10] analizaron la postura de jóvenes jugadores de tenis de mesa y concluyeron que, en general, los jóvenes tienen mala postura, pero no pudieron relacionar la postura con el nivel de práctica deportiva. Macedo y col [15] evaluaron el efecto de un tratamiento para la lumbalgia en ciclistas y observaron alguna variación postural después de su aplicación.

Existe una tendencia creciente en el entrenamiento de fuerza y condición física para identificar patrones de movimiento defectuosos de los deportistas. La valoración funcional previa permite individualizar los ejercicios en función de las necesidades e incorporal los ejercicios compensatorios adecuados para la prevención de la lesión. El objetivo de este estudio piloto es el de analizar la postura de pie en deportistas.

\section{Materiales y métodos}

Participaron en el estudio 7 estudiantes (6 hombres y 1 mujer) de diferentes modalidades deportivas, con una media de $24.6 \pm 0.5$ años, $72 \pm 16,8 \mathrm{~kg}$ de peso y $174 \pm 13.1 \mathrm{~cm}$ de altura. Todos cumplieron con los criterios de inclusión siguientes: (1) No estar lesionado en el momento de la valoración. (2) Si han estado lesionados, tienen que estar recuperados i haber cursado la actividad deportiva un mínimo de dos meses. (3) Los sujetos no pueden estar tomando antidepresivos. El estudio fue aprobado por el Comité de Ética de la Universitat de Vic.

Para la valoración de la postura se utilizó una cámara digital (Canon EOS 400) situada sobre un trípode a una distancia de 3 metros de la zona delimitada para la posición del sujeto siguiendo las instrucciones de Ferreira y 
col [7]. El espacio fue calibrado mediante una plomada. Las fotografías fueron tomadas de cuerpo entero en el plano frontal (anterior y posterior) y sagital (lados derecho e izquierdo). La posición de los pies estaba debidamente señalizada. Para que la postura fuera lo más natural posible se pidió a los sujetos que cerraran los ojos durante su colocación, llevara la cabeza a la posición neutra, abriera los ojos y mantuviera la mirada fija en un punto. Este procedimiento se realizó para las cuatro posiciones evaluadas. Los puntos de referencia anatómicos fueron marcados mediante bolas de poliestireno expandido de $10 \mathrm{~mm}$ de diámetro siguiendo el protocolo de Ferreira y col [7]. Las fotografías fueron procesadas mediante el software de análisis postural SAPO (Sao Paulo) obteniendo los siguientes parámetros mecánicos (Figura 1): Vista anterior: (1A) alineación horizontal de cabeza, (2A) alineación horizontal acromion, (3A) alineación horizontal crestas ilíacas antero-superiores (ASIS), (4A) diferencia longitud extremidades inferiores, (5A) alineación horizontal rodillas, (6A-7A) ángulo Q derecho e izquierdo. Vista posterior: (1P) Asimetría horizontal espina de la escápula - proceso espinoso vértebra torácico 3, (2P-3P) ángulo pierna - retropié derecho e izquierdo. Vista lateral (igual para los dos lados, derecho e izquierdo): (1L) alineación horizontal de la cabeza, (2L) alineación vertical cabeza, (3L) alineación vertical del tronco, $(4 \mathrm{~L})$ ángulo tronco - piernas, $(5 \mathrm{~L})$ alineación vertical del cuerpo, (6L) Alineación horizontal cadera, (7L) ángulo flexión rodilla, (8L) ángulo horizontal pierna.

\section{Resultados}

En la tabla 1 se pueden observar los resultados para el plano frontal. Las desviaciones medias respecto a la línea horizontal son de máximo $1,5^{\circ}$. Para las desviaciones horizontales se ha tomado el valor absoluto, pues no todos los sujetos presentan desviación hacia el mismo lado corporal. La alineación de referencia es de $0^{\circ}$ [1]. Los valores promedios para el ángulo Q están un poco por debajo de los valores de referencia propuestos por Horton y Hall [16]. Un sujeto presenta valores negativos del ángulo, con un varo y una rotación externa de rodilla acentuados. El valor de los

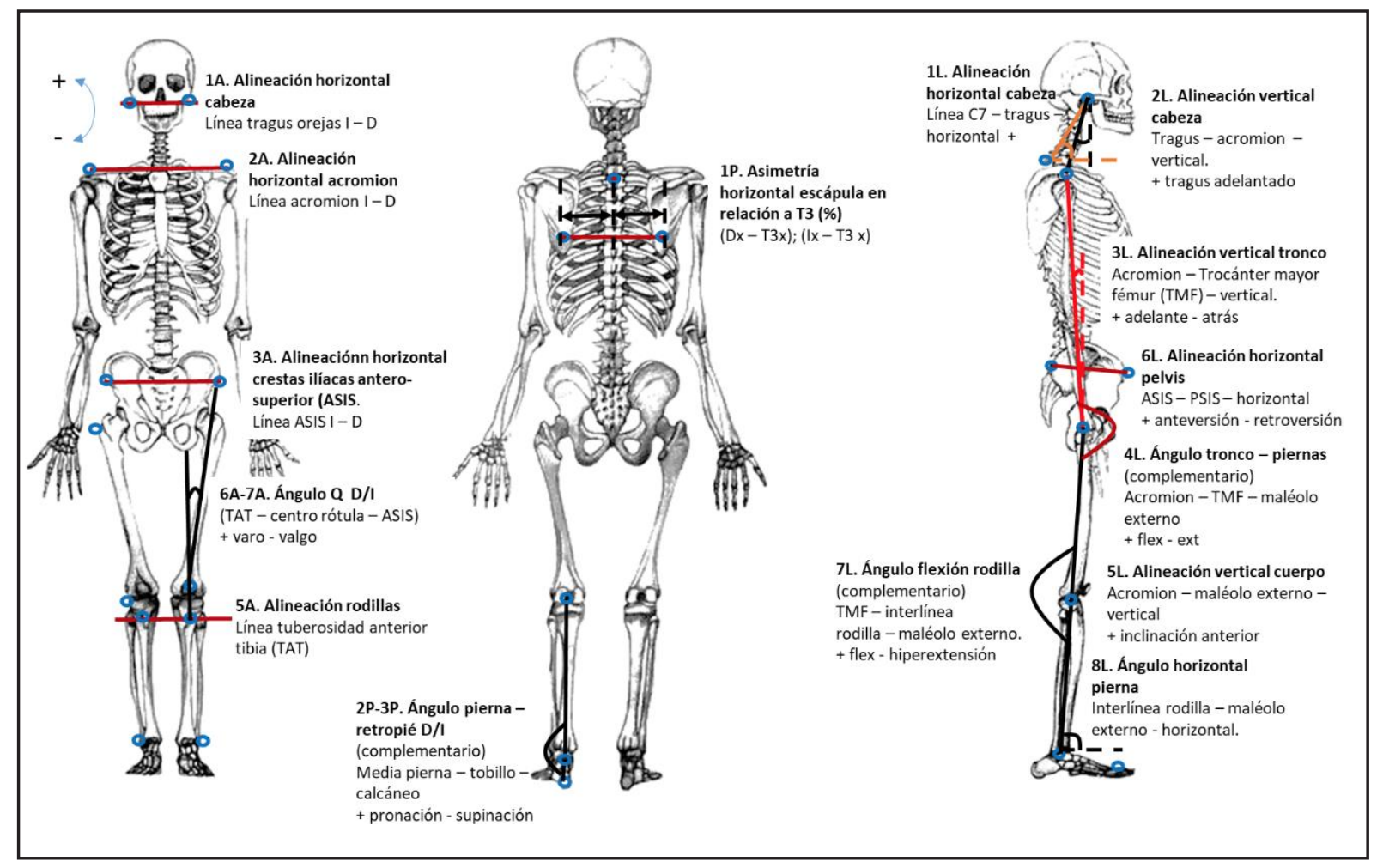

Figura 1. Parámetros, referencias anatómicas y convenciones. 
ángulos Q no es simétrico para las dos extremidades, aunque la diferencia es un poco menor que la encontrada $\left(4^{\circ}\right)$ por Livingston y Mandigo [17].

Para la vista posterior, encontramos una leve asimetría entre escapulas $(4,3 \%)$, siendo el valor máximo de $13,5 \%$ para uno de los sujetos. El promedio del ángulo del retropié también está dentro de los valores de referencia [18].

Para el plano sagital (Tabla 2) se ha realizado el promedio de ambos lados derecho e izquierdo. Cabe destacar la diferencia en la alineación vertical de la cabeza que es de promedio de $18^{\circ}$. Según Kendall y col [1], tragus y acromion tendrían que estar alineados. La alineación horizontal de la cabeza $\left(48,9^{\circ}\right)$ es parecido a los valores encontrados en la literatura $\left(49^{\circ}-51^{\circ}\right)$ [19]. Tanto el tronco, como el total del cuerpo muestran una ligera inclinación hacia adelante, también se observa una ligera hiperextensión en la cadera que podría afectar las curvaturas lumbares. En posición de pie la rodilla presenta una ligera

\begin{tabular}{|c|c|c|c|c|c|}
\hline Variable & Referencia & Promedio & DS & Min & Max \\
\hline *Alineación H cabeza $\left(^{\circ}\right)$ & $0^{\mathrm{o}}$ & 1,5 & 0,8 & 0,4 & 2,4 \\
\hline${ }^{\star}$ Alineación $\mathrm{H}$ hombros $\left({ }^{\circ}\right)$ & $0^{\mathrm{o}}$ & 0,9 & 1,0 & 0,2 & 2,9 \\
\hline${ }^{*}$ Alineación H ASIS $\left(^{\circ}\right)$ & $0^{\mathrm{o}}$ & 1,5 & 1,1 & 0,0 & 3,4 \\
\hline${ }^{\star}$ Diferencia longitud EEII $(\mathrm{cm})$ & $0^{\mathrm{o}}$ & 0,8 & 0,8 & 0,0 & 1,9 \\
\hline${ }^{\star}$ Alineación $\mathrm{H}$ rodillas $\left(^{\circ}\right)$ & $0^{\mathrm{O}}$ & 0,9 & 0,7 & 0,0 & 2,3 \\
\hline $\mathrm{D}$ & $\approx 8-14^{\mathrm{o}} \mathrm{H}$ & 5,6 & 4,1 & $-0,6$ & 11,1 \\
\hline Ángulo Q $\left(^{\circ}\right) \quad$ I & $\approx 11-20^{\circ} \mathrm{H}$ & 7,3 & 5,3 & $-1,9$ & 13,5 \\
\hline${ }^{*}$ Diferencia & $0^{\mathrm{O}}$ & 3,8 & 2,1 & 0,7 & 5,9 \\
\hline Asimetria H escapulas -T3 (\%) & $0 \%$ & 4,3 & 4,3 & 0,0 & 13,1 \\
\hline $\mathrm{D}$ & $0^{\mathrm{O}}-5^{\mathrm{O}}$ & 2,8 & 1,4 & 1,1 & 4,9 \\
\hline Ángulo retropie $\left(^{\circ}\right) \quad$ I & $0^{2}-5^{2}$ & 4,3 & 3,7 & 0,4 & 10,2 \\
\hline${ }^{\star}$ Diferencia & & 2,7 & 3,0 & 0,1 & 7,5 \\
\hline
\end{tabular}

Tabla 1. Promedio, desviación estándar y rango de los valores del plano frontal.

\begin{tabular}{|c|c|c|c|c|c|c|}
\hline Variable & Referencia & Promedio & DS & Min & $\operatorname{Max}$ & Diferencia D-I \\
\hline Alineación H cabeza $\left(^{\circ}\right)$ & & 48,8 & 4,0 & 43,3 & 54,8 & 1,7 \\
\hline Alineación V cabeza $\left({ }^{\circ}\right)$ & $0^{\circ}$ & 18,1 & 5,8 & 9,1 & 32,1 & $-5,5$ \\
\hline Alineación $\mathrm{V}$ tronco $\left({ }^{\circ}\right)$ & $0^{\circ}$ & 0,6 & 1,9 & $-2,8$ & 3,5 & 0,3 \\
\hline Ángulo cadera $\left(^{\circ}\right)$ & $0^{\circ}$ & $-2,5$ & 3,9 & $-9,6$ & 3,3 & $-0,7$ \\
\hline Alineación V cuerpo $\left(^{\circ}\right)$ & $0^{\circ}$ & 2,5 & 1,3 & 0,3 & 5,1 & 0,8 \\
\hline Alineación H pelvis $\left(^{\circ}\right)$ & & $-10,4$ & 7,3 & $-18,5$ & 2,5 & 0,5 \\
\hline Ángulo flexión rodilla $\left({ }^{\circ}\right)$ & $0^{\mathrm{O}}$ & 1,3 & 3,7 & $-5,5$ & 7,0 & 0,5 \\
\hline Ángulo H pierna $\left({ }^{\circ}\right)$ & & 85,6 & 2,2 & 81,6 & 89,6 & $-1,5$ \\
\hline
\end{tabular}

Tabla 2. Promedio, desviación estándar, rango y diferencia derecha e izquierda de los valores del plano sagital. 
flexión y la cadera presenta unos valores de anteversión de $10,4^{\circ}$ indicando hiperlordosis lumbar. Según Kritz y Cronin [20] las ventajas de hiperlordosis y anteversión pélvica resultarían en aplicar fuerzas durante más tiempo incrementando así el impulse mecánico, pero el incremento de la lordosis lumbar también está relacionado con un mayor dolor en esta zona corporal.

\section{Conclusiones}

En el estudio piloto se pueden observar ligeras modificaciones respecto del patrón de postura considerado ideal. En un futuro se pretende desarrollar una base de datos más extensa, ampliar la información con una anamnesis de lesiones y otros test de valoración funcional. Sería interesante poder establecer alguna correlación e identificar patrones de postura considerados factores de riesgo para padecer una lesión, así como conocer si se producen modificaciones de la postura en función del tipo de modalidad deportiva practicada.

\section{Agradecimientos}

Los autores agradecen la participación de los estudiantes de CAFE de la UVic.

\section{Referencias}

1. Kendall FP, McCreary EK, Provance PG, Rodgers MM, Romani WA. Muscles: Testing and function with posture and pain. Baltimore: Lippincott Williams \& Wilkins. 2005.

2. Pownall PJ, Moran RW, Stewart AM. Consistency of standing and seated posture of asymptomatic male adults over a one-week interval: A digital camera analysis of multiple landmarks. Int J Osteopath Med 2008; 11; 43-51.

3. Rosário JLP. Photographic analysis of human posture: A literature Review, J Bodyw Mov Ther, 2014; 18; 56-61.

4. Fortin C, Feldman DE, Cheriet F, Labelle $H$. Clinical methods for quantifying posture: a literature review. Disabil Rehabil 2011; 33; 367-83.

5. Zonnenberg AJI, Van Maanen CJ, Elvers JWH, Oostendrop RAB. Intra/interrater reliability of measurements on body posture photographs. Cranio 1996; 14; 326-331.

6. McEvoy MP, Grimmer K. Reliabillity of upright posture measurements in primary school chil- dren. BMC Musculoskelet Disord 2005; 6; 35.

7. Ferreira EAG, Duarte M, Maldonado EP, Burke TN, Marques AP. Postural assessment software (PAS/SAPO): validation and reliability. Clinics 2010; 65; 75-81.

8. Dunk NM, Lalonde J, Callaghan JP. Implications for the use of postural analysis as a clinical diagnostic tool: reliability of quantifying upright standing spinal postures from photographic images. J Manipulative Physiol Ther 2005; 28; 386-392.

9. Pausic J, Pedisic Z, Dizdar D. Reliability of a photographic method for assessing standing posture of elementary school students. J Manipulative Physiol Ther 2010; 33; 425-443.

10. Munivrana G, Pausic J, Kondric M. The incidence of improper postural alignment due to the influence of long-term table tennis training. Kinesiologia Slovenica, 2011; 17; 47-58.

11. Puglisi F, Donati P, Marini M, Romero L, Scidone S, Ferrari R. A photographic method for multi-plane assessment of adolescent posture. Ital J Anat Embryol 2014; 119; 241-249.

12. Normand MC, Descarreaux M, Harrison DD, Harrison DE, Perron DL, Ferrantelli JR, Janik T. three dimensional evaluation of posture in standing with the PosturePrint: an intra- and inter-examiner reliability study. Chiropr Osteopat 2007; 15; 5.

13. Rosário JLP, Nakashima IY, Rizopoulos K, Kostopoulos D, Marques AP. Improving posture: Comparing segmental stretch and muscular chains therapy. Clinical Chiropractic 2012; 15; 121-128.

14. Sinzato CR, Taciro C, Pio CA, Toledo AMT, Cardoso JR, Carregaro RL. Effects of 20 sessions of Pilates method on postural alignment and flexibility of young women: pilot study. Fisioter Pesq 2013; 20; 143-150.

15. Macedo RMB, Ricieri DV, Ulbricht L, Preis C, Duarte JM, Bertassoni L. Análise cinemática 2d da postura ortostática de ciclistas lombálgicos. Uniandrade 2013; 14; 7-23.

16. Horton MG, Hall TL. Quadriceps femoris muscle angle: normative values and relationships with gender and selected skeletal measures. Phys Ther 1989; 69; 897-901.

17. Livingston AV, Mandigo JL. Bilateral Q angle asymmetry and anterior knee pain syndrome. Clin. Biomech, 1999; 14; 7-23.

18. Eng JJ, Pierrynowski MR. The effect of soft foot orthotics on three-dimensional lower-limb kinematics during walking and running. Phys Ther 1994; 74; 836-44.

19. Christie HJ, Kumar S, Warren SA. Postural aberrations in low back pain. Arch Phys Med Rehabil 1995; 76; 218-224.

20. Kritz MF, Cronin J. Static posture assessment screen of athletes: Benefits and considerations. SCJ 2008; 5; 18-27. 